\title{
UK NEQAS for leucocyte immunophenotyping: the first 10 years
}

\author{
J T Reilly, D Barnett
}

\begin{abstract}
In the past decade, cellular immunophenotyping has become a new discipline in diagnostic haematology and immunology, and is invaluable in the rapid diagnosis of leukaemia and monitoring disease progression in human immunodeficiency virus infected individuals. The introduction of bench top flow cytometers has meant that immunophenotyping is now also used for the quantitation of $\mathrm{CD}^{+}$ peripheral blood stem cells (PBSCs) to ensure the correct timing and adequacy of haematopoietic progenitor cell harvests. Furthermore, flow cytometry has become an important tool for the counting of leucocytes in blood components after leucocyte depletion. Because this new discipline is now such a major diagnostic and prognostic tool in the clinical arena, its use must be subject to both internal and external quality control. Such a requirement was first recognised as early as 1986 when an Inter-Regional Quality Assessment Scheme (IRQAS) was initiated for laboratories that undertook the immunocytochemical diagnosis of leukaemia using the alkaline phosphates antialkaline phosphatase technique. This programme began with around $25 \mathrm{UK}$ laboratories. In 1990, after the introduction of two more programmes (one for leukaemia diagnosis using UV microscopy and latterly flow cytometry, and one for the enumeration of $\mathrm{CD}^{+} \mathrm{T}$ cells) the IRQAS achieved UK National External Quality Assessment Scheme (UK NEQAS) status and changed its title to UK
\end{abstract} NEQAS for Leucocyte Immunophenotyping. In the past decade the once small IRQAS programme has evolved into the largest international scheme of its kind, providing EQA to over 650 laboratories world wide for leukaemia immunophenotyping, lymphocyte subset analysis, PBSCs, and more recently low level leucocyte counting. Over the years, this EQA programme has highlighted important problems, such as the inappropriate use of fluorochromes and antibody titre, and the identification of effective gating strategies, all of which have contributed directly to the high interlaboratory variations seen in cellular immunophenotyping. Furthermore, particularly in absolute counting of lymphocyte subsets, PBSCs, and the enumeration of low numbers of leucocytes, UK NEQAS for Leucocyte Immunophenotyping programmes have been instrumental in highlighting the differences that occur between single and dual platform flow cytometric technologies. As a result of these findings, UK NEQAS for Leucocyte Immunophenotyping has helped to reduce the variation seen on an interlaboratory basis and enabled greater standardisation both in the UK and internationally. These advances have been attributable to the development, by UK NEQAS for Leucocyte Immunophenotyping, of a unique whole blood stabilising process that ensures the retention of the physical characteristics (both light scatter and antigenic profile) required of cells to ensure successful cellular immunophenotyping. This major technological advancement has enabled the distribution of specimens for EQA purposes on a global scale that have minimal matrix effect and behave in a manner identical to fresh blood for several months after stabilisation.

(F Clin Pathol 2001;54:508-511)

Keywords: leucocyte immunophenotyping; leukaemia; human immunodeficiency virus infection; flow cytometry; guidelines

Since its modest origins as an Inter-Regional Quality Assessment Scheme (IRQAS) for Immunocytochemistry, the UK National External Quality Assessment Scheme (NEQAS) for Leucocyte Immunophenotyping has progressively evolved over the past decade to become the largest international scheme of its kind, incorporating assessment programmes for leukaemia immunophenotyping (immunocytochemistry and immunofluorescence) as well as for $\mathrm{CD}^{+} \mathrm{T}$ cell, $\mathrm{CD} 34^{+}$peripheral blood stem cell, and low level leucocyte enumeration. A pivotal factor in the genesis of the scheme has been the development of a unique stabilising process that ensures the retention of leucocyte light scatter and immunological staining characteristics for up to 300 days. ${ }^{1}$ Previously, EQA programmes have used either fresh whole blood or frozen cells. The instability of such analytes means that samples need to be shipped by express courier, making the price of transportation one of the heaviest cost burdens in EQA. More importantly, wide coefficients of variation (CVs) are seen with the use of fresh whole blood and frozen cells, a fact that may potentially mask the identification of crucial elements for satisfactory laboratory performance. This article aims to review the findings from the first 10 years of activity of UK 
NEQAS and to highlight briefly the areas that will need to be dealt with in the near future.

\section{Leukaemia immunophenotyping}

The commercial availability of a very wide range of specific monoclonal antibodies has made interlaboratory reproducibility, and hence external quality control, difficult. A survey of routine UK laboratories in 1989 revealed a total of 86 antibodies being used as front line reagents for leukaemia diagnosis. Furthermore, the use of monoclonal antimyeloperoxidase antibody, probably the single most informative reagent, ${ }^{2}$ was frequently omitted. The publication of the British Committee for Standards in Haematology guidelines, which included recommended minimum antibody panels for both acute and chronic leukaemias, ${ }^{34}$ has resulted in a greater degree of standardisation in this area of UK practice. Many problems still remain, however, including the lack of standardisation of analysis techniques (for example, direct or indirect immunofluorescence, one or two stage immunocytochemistry, and the use of different flow cytometric gating strategies), as well as the use of different antibody sources and dilutions, fluorochrome conjugates, and lysing and fixative reagents.

UK NEQAS has highlighted the effect that different fixatives may have on immunocytochemical antigen detection. Indeed, we have shown repeatedly that many antigens are best preserved by using cold pure acetone fixation and that laboratories using formalin containing fixatives may miss these diagnostically important antigens. Furthermore, cells labelled directly with fluorescein isothiocyanate (FITC) conjugated antibodies will not fluoresce as brightly as those stained using indirect FITC methods, a fact of practical importance when antigen expression is low. Phycoerythrin (PE) and the newer fluorochromes (for example, tandem colour fluorochromes such as $\mathrm{PE} / \mathrm{Cy} 5$ ) have a much higher quantum yield than FITC, thus increasing sensitivity. As a result, significant differences have been documented for samples analysed with FITC or PE conjugated antibodies. For example, in a UK NEQAS survey investigating CD13 detection, eight of 24 laboratories that used FITC conjugated antibodies obtained values of $<50 \%$ (mean, $58 \%$ ), of which three were negative results. In contrast, all 12 laboratories using PE conjugated reagents obtained values $>50 \%$ (mean, $77 \%$ ). Therefore, PE conjugates, or tandem colour fluorochromes (such as PE/Cy5), should be used for single colour analysis. For multicolour analysis, the more sensitive fluorochrome conjugated antibody should be used for detecting the weaker antigen, typically CD13, CD19, or CD33, whereas strongly expressed antigens (such as CD45 or HLADR) can be detected using FITC or peridin chlorophyll protein (PerCP). Data from UK NEQAS have also revealed that UK laboratories use a wide variety of antibody dilutions, even when antibodies are obtained from the same source; a fact that has resulted in demonstrable prozone effects and non-specific binding when antigens are identified by immunocytochemistry. Ideally the "titre value" should be determined for every antibody, especially when investigating suspected non-specific binding. ${ }^{5}$

EQA schemes for the laboratory diagnosis of leukaemia will need to evolve, as recent advances in reagents and techniques become routine practice. For example, the past five years have witnessed the use of additional antibodies (such as anti-CD79a/b, anti-CD117, and anti-cyclin D1), double and triple immunostaining, reagents and techniques for permeabilising cells to permit the detection of nuclear and cytoplasmic antigens, gating strategies using CD45 to allow selected cell population analysis, and the quantitation of surface and cellular antigens. Indeed, the availability of new diagnostically important antibodies has already led to a revision of current guidelines. $^{6}$

\section{$\mathrm{CD}^{+} \mathrm{T}$ cell enumeration}

Accurate absolute $\mathrm{CD} 4^{+}$cell counts, as well as their percentage values, are two crucial assays used for the monitoring of human immunodeficiency virus (HIV) infection. They are required for the following: (1) to assess the degree of immune deterioration and rate of progression towards AIDS (defined as a $\mathrm{CD} 4^{+}$ lymphocyte count of $<0.2 \times 10^{9} /$ litre or $<14 \%$ ), (2) to group HIV seropositive patients into cohorts according their baseline $\mathrm{CD}^{+}$ counts before starting treatment, (3) to determine the appropriate time for prophylaxis of opportunistic infections, and (4) to monitor the efficacy of antiretroviral and/or interleukin 2 treatment. Therefore, there is a clinical need for the accurate and precise enumeration of $\mathrm{CD} 4^{+} \mathrm{T}$ cells. In the early 1980 s, such estimations were undertaken using Ficoll density separated lymphocytes and ultraviolet microscopy. However, recent technological advances have rendered such methods obsolete and the recommended approach is now multiparametric lymphocyte immunophenotyping, using whole blood and incorporating one of three gating strategies, namely: $\mathrm{T}$ gating, lineage gating, or CD 45/side scatter gating. ${ }^{7} \mathrm{UK}$ NEQAS has shown, by using stabilised whole blood and strict adherence to gating strategies, that individual laboratory results for a given issue can be tightly distributed about the mean, as defined by a low SD value. Most CV values have been $<10 \%$ for each of the three parameters, namely: $\mathrm{CD}_{3}^{+}, \mathrm{CD}^{+} \mathrm{CD}^{+}$, and $\mathrm{CD}^{+} \mathrm{CD}^{+}$. In general, absolute $\mathrm{T}$ cell counts are calculated from the absolute lymphocyte count and the percentage $T$ cell subset value; a method termed the "dual platform approach". However, recent reports have highlighted the variability in absolute $T$ cell counts as a result of using different haematology analysers. ${ }^{8}$ To overcome this problem, single platform flow cytometers capable of generating absolute $\mathrm{T}$ cell counts have been developed, which depend on either precision fluidics or microbead technology. We have shown that consistently lower 
CVs are obtained using such technology and have suggested that this approach is the method of choice. ${ }^{9}$

\section{$\mathrm{CD}^{+} 4^{+}$stem cells}

Clinicians routinely rely on $\mathrm{CD} 34^{+}$cell quantitation by flow cytometry, as a marker of pluripotential stem cells, to ensure the correct timing and adequacy of haematopoietic progenitor cell harvests. The assessment of progenitors in colony forming assays is limited by lack of reproducibility and prolonged assay time. A minimum threshold dose of $2 \times 10^{6}$ $\mathrm{CD} 34^{+}$cells/kg has been found in multiple clinical settings to result in adequate engraftment. ${ }^{10}$ However, no proposed guidelines on methodology have been universally accepted. For example, a variety of flow cytometric gating strategies for $\mathrm{CD}_{3} 4^{+}$cell enumeration have been developed, based primarily upon the detection of total $\mathrm{CD} 34^{+}$, or $\mathrm{CD} 34^{+} \mathrm{CD} 45^{\mathrm{dim}}$ cells. In addition, there is great variation in the choice of monoclonal antibody, fluorochrome, and the lysing solution used. Furthermore, the absolute enumeration of $\mathrm{CD} 34^{+}$cells can be determined using either a dual or single platform approach. In an attempt to deal with these problems of standardisation, several national EQA programmes and international workshops have been set up during the past five years (reviewed by Barnett et al). ${ }^{7}$ However, widely varying interlaboratory $\mathrm{CVs}$ were documented (CVs greater than 100\%), mainly because of the use of fresh or cryopreserved specimens. ${ }^{11}$ This view is supported by the findings of a recent Australian study, which found a pronounced reduction of interlaboratory CVs when only list mode data (multiparametric data stored after sample analysis) were analysed. ${ }^{12}$ The same study also demonstrated that gating strategies contributed to result variability.

In response to such findings, UK NEQAS initiated an EQA scheme in 1994, using stabilised whole blood samples, which currently involves 132 laboratories in 22 countries. Several crucial analytical variables have been highlighted. First, there was a pronounced variation in the number of $\mathrm{CD}_{3} 4^{+}$cells counted routinely (10 000 to 1.2 million), with several laboratories collecting significantly fewer than 50 000. A reduction in the number of events for each test will reduce the reliability of the estimation to unacceptable levels. Given the fact that the standard error of the number of positive cells is given by the square root of the number of positive cells, the larger the acquisition the lower the CV. In addition, most centres used a fixed volume for analysis, without taking into account the cell count. It has been stated that a minimum of 100 CD $34^{+}$ events should be collected from at least 75000 CD45 events to maintain precision and also to ensure a methodological CV of $<10 \% .^{13}$ Second, different lysing solutions and methodologies were used for routine analysis. It is well documented that systems using "lyse no wash" or "no lyse no wash" techniques have reduced variability and tighter CVs for $\mathrm{CD}^{+} \mathrm{T}$ cell enumeration. UK NEQAS has confirmed this observation for $\mathrm{CD} 34^{+}$cell enumeration and found that laboratories using lyse no wash systems returned approximately 20\% higher CD $34^{+}$counts, suggesting that cells are lost during the washing process. ${ }^{7}$ It should also be stressed that certain lysing reagents may reduce antigen expression and therefore be a source of additional variability. ${ }^{14}$ It is possible that the reduction of antigen density caused by a particular lysing reagent, coupled with the use of FITC conjugated antibodies and a lyse no wash technique could result in peripheral blood stem cells being significantly underestimated. Lastly, the mean interlaboratory CV using non-standardised, single platform approaches was significantly lower $(18.6 \%)$ when compared with laboratories using dual platform technology $(28.6 \%) .{ }^{9}$ In conclusion, UK NEQAS has demonstrated that interlaboratory CVs can, on an international scale, be reduced to less than $25 \%$ without using specified gating criteria. However, our findings indicate that further improvements are possible if standardised protocols are adopted and highlight the urgent need for internationally agreed consensus protocols. These problems have recently been dealt with in the UK with the publication of guidelines by the British Committee for Standards in Haematology and their impact on UK laboratory performance is awaited with interest. $^{15}$

\section{Low level leucocyte counting}

The use of leucocyte depleted blood products has been shown to reduce, or prevent, adverse transfusion reactions, transfusion related bacterial sepsis, febrile reactions, human major histocompatibility complex (HLA) antigen alloimmunisation, and the transmission of viruses. Furthermore, the emergence of variant Creutzfeldt-Jakob Disease (vCJD) in the UK, coupled with increasing evidence that the distribution of this disease in human tissue may be different to that of the classic form of CJD, has raised questions regarding the safety of UK plasma products. Recent guidelines suggest that blood product units should have $<5 \times 10^{8}$ leucocytes to prevent transfusion febrile reactions and $<5 \times 10^{6}$ to prevent alloimmunisation and the transmission of cytomegalovirus. ${ }^{16}$ This last value has recently been adopted by the UK transfusion services and currently all issued blood products are required to contain leucocyte counts of fewer than $5 \times 10^{6}$. As a result, UK NEQAS for Leucocyte Immunophenotyping, in collaboration with the UK transfusion services, initiated an EQA programme for low level leucocyte counting in 1998 that has now expanded to involve 30 sites in the UK and mainland Europe. The programme has highlighted the current variability in performance, especially within the critical range of 5-30 cells/ $\mu 1$ (which equates approximately to blood products with leucocyte counts of $<5 \times 10^{6}$ ). Several factors have been identified, including analytical technology, gate placement, and the method of leucocyte identification. For example, the Nageotte counting chamber gave the highest interlaboratory CVs (mean, $61.8 \%$ ) when compared with 
flow cytometric based analyses (mean, 29.5\%), and significant variability existed within the flow cytometric user group, particularly in relation to analysis region placement. In addition, laboratories that used commercial nuclear stains had consistently lower CVs than those using "in house" propidium iodine methods. Importantly, these analytical differences resulted in several centres reporting a given sample as being "out of consensus", which would have led theoretically to blood components being inappropriately released. As a result, UK NEQAS recommends that a standardised protocol should be used for the routine validation of leucocyte depleted blood products that incorporates targeted training, and the use of flow cytometric analysis with the adoption of common gating strategies and nuclear staining reagents.

\section{The next 10 years?}

The past 10 years have seen EQA become an integral part of the everyday working routine of cell marker laboratories. Indeed, the rise in participants seen over this period underlines the emphasis that laboratories worldwide place upon such a service. As shown previously, UK NEQAS for Leucocyte Immunophenotyping has kept pace with these needs and has also ensured that developments are introduced in a timely fashion. But what of the next 10 years? Ideally, external quality assessment of leukaemia immunophenotyping should evaluate all aspects of the diagnostic process, including antibody panel selection, technical analysis (incorporating sample processing), and data acquisition, analysis, and interpretation. To date, however, most EQA schemes have focused on assessing technical performance ${ }^{17-19}$ and, with the exception of the Dutch programme ${ }^{20}$ have not yet assessed data interpretation. Clearly, this area needs to be considered in the future because individual diagnostic performance will probably form an increasingly important aspect of clinical governance. Planned UK NEQAS projects include the development of a "daily run" control standard for low level leucocyte enumeration, a short term fixative for use with clinical samples, and the development of stable material to enable the introduction of an EQA scheme for paroxysmal nocturnal haemoglobinuria. Specific projects are also under way to standardise clinical flow cytometry on an international basis. This project has been boosted by financial support from the European Commission and will enable UK NEQAS to collaborate with partners from Portugal, Italy, Germany,
Holland, Greece, Sweden, and Spain to develop stable European reference preparations for use in flow cytometry. Once developed, the reference materials will be deposited at national facilities and will be available for validating new techniques. In the final year of the project (2003) the group will attempt to develop an infrastructure for a European wide network of EQA schemes.

1 Barnett D, Granger V, Mayr P, et al. Preparation and stabilisation of cells. Patent No. WO 95/01796, 1995:1-31.

2 Storr J, Dolan G, Coustan-Smith E, et al. Value of monoclonal anti-myeloperoxidase (MP07) for the diagnosis of acute leukaemia. $\mathcal{F}$ Clin Pathol 1990;43:847-9.

3 Rowan RM, Bain BJ, England JM, et al. Immunophenotyping in the diagnosis of acute leukaemias. General ing in the diagnosis of acute leukaemias. General 777-81.

4 Rowan RM, Bain BJ, England JM, et al. Immunophenotyping in chronic (mature) lymphoproliferative disorders. General haematology task force of BCSH. 7 Clin Pathol 1994;47:871-5

5 Reilly JT. Use and evaluation of leucocyte monoclonal antibodies in the diagnostic laboratory: a review. Clin Lab Haem 1996;18:1-5.

6 Bain B, Barnett D, Linch DC, et al. Revised guidelines on immunophenotyping in acute and chronic lymphoproliferative disorders. Clin Lab Haematol 2001 [In press.]

7 Barnett D, Granger V, Storie I, et al. Quality assessment of $\mathrm{CD} 34^{+}$stem cell enumeration: experience of the United Kingdom National External Quality Assessment Scheme (UKNEQAS) using a stable whole blood preparation. $\mathrm{Br} f$ Haematol 1998;102:553-65.

8 Robinson G, Morgan L, Evans M, et al. Effect of type of haematology analyser on CD4 count. Lancet 1992;340: 485 .

9 Barnett D, Granger V, Whitby L, et al. Absolute $\mathrm{CD}^{+}$ T-lymphocyte and $\mathrm{CD} 34^{+}$stem cell counts by singleplatform flow cytometry: the way forward. $\mathrm{Br} \mathcal{F}$ Haematol 1999;106:1059-62.

10 Krause DS, Fackler MJ, Civin CI, et al. CD34: structure, biology, and clinical utility. Blood 1996;87:1-13.

11 Gratama JW, Kraan J, Levering W, et al. Analysis of variation in results of $\mathrm{CD} 34+$ hematopoietic progenitor cell enumeration in a multicenter study. Cytometry 1997;30: 109-17.

12 Chang A, Ma D. The influence of flow cytometric gating strategy on the standardization of CD34+ cell quantitation: an Australian multi-centre study. 7 Hematother 1996;5: 605-16.

13 Sutherland DR, Anderson L, Keeney M, et al. The ISHAGE guidelines for $\mathrm{CD} 34^{+}$cell determination by flow cytometry. International Society of Hematotherapy and Graft Engineering. F Hematother 1996;5:209-10.

14 Macey MG, McCarthy DA, van Agthoven A, et al. How should CD $34^{+}$cells be analysed? A study of three classes of antibody and five leucocyte preparation procedures. $\mathcal{F}$ Immunol Methods 1997;204:175-88.

15 Barnett D, Janossy G, Lubenko A, et al. Guideline for the flow cytometric enumeration of $\mathrm{CD} 34^{+}$haematopoietic stem cells 1999. Clin Lab Haematol 1999;21:301-8.

16 British Committee for Standards in Haematology. Guidelines on the clinical use of leucocyte-depleted blood lines on the clinical use of leucocy
components. Transf Med 1998;8:59-71.

17 Forrest MJ, Barnett D. Laboratory control of immunocytochemistry. Eur f Haematol 1989;42:67-71.

18 Hassett J, Parker J. Laboratory practices in reporting flow cytometry phenotyping results for leukemia/lymphoma specimens: results of a survey. Cytometry 1995;22:264-81.

19 Homburger HA, Rosenstock W, Paxton H, et al. Assessment of interlaboratory variability of immunophneotyping. Results of the College of American Pathologists flow cytometry survey. Ann N Y Acad Sci 1993;677:43-9.

20 van't Veer MB, Kluin-Nelemans JC, van der Schoot CE, et al. Quality assessment of immunological marker analysis and the immunological diagnosis in leukaemia and lymphoma: a multi-centre study. Dutch Cooperative Study Group on Immunophenotyping of Leukemias and Lymphomas (SIHON). Br f Haematol 1992;80:458-65. 